\title{
Spatial variability of the sea-ice radiation budget and its effect on aggregate-area fluxes
}

\author{
Xuanji Wang, ${ }^{1}$ Jeffrey R. Key ${ }^{2}$ \\ ${ }^{1}$ Cooperative Institute for Meteorological Satellite Studies, University of Wisconsin, 1225 West Dayton Street, Madison, WI 53706-1490, U.S.A. \\ ${ }^{2}$ Office of Research and Applications, National Environmental Satellite Data and Information Service, National Oceanic and Atmospheric \\ Administration, University of Wisconsin, 1225 West Dayton Street, Madison, WI 53706-1490, U.S.A.
}

\begin{abstract}
The spatial and temporal variability of surface, cloud and radiative properties of sea ice are examined using new satellite-derived products. Downwelling short- and longwave fluxes exhibit temporal correlation over about 180 days, but cloud optical depth and cloud fraction show almost no correlation over time. The spatial variance of surface properties is shown to increase much less rapidly than that of cloud properties. The effect of small-scale inhomogeneity in surface and cloud properties on the calculation of radiative fluxes at ice- and climate-model gridscales is also investigated. Annual mean differences between gridcell fluxes computed from average surface and cloud properties and averages of pixel-by-pixel fluxes are $9.46 \%$ for the downwelling shortwave flux and $-7.04 \%$ for the longwave flux. Therefore, using mean surface and cloud properties to compute surface radiative fluxes in a gridcell results in an overestimate of the shortwave flux and an underestimate of the longwave flux. Model sensitivity studies show that such biases may result in substantial errors in modeled ice thickness. Clearly, the sub-gridscale inhomogeneity of surface and atmospheric properties must be considered when estimating aggregate-area fluxes in sea-ice and climate models.
\end{abstract}

\section{INTRODUGTION}

Numerical models of weather, climate and sea ice typically use radiative-flux parameterizations that are based on the assumption that the underlying surface is homogeneous. Recent studies have shown that this assumption can result in errors in estimating surface radiation and turbulent fluxes because some atmospheric and surface properties are non-linear with respect to surface radiation fluxes (Mahrt, 1987; Sellers, 1991; Friedl, 1996). How large are the errors in radiative fluxes computed from area-average surface and cloud properties? The magnitude of the error depends on the degree of non-linearity in the relationship and on the spatial variability of the parameters. For example, the relationship between cloud optical depth and shortwave radiation is highly non-linear and the spatial variability of optical depth is often large. Conversely, surface albedo and shortwave radiation are linearly related and, except for the summer months, the surface albedo of sea ice is relatively homogeneous over large areas.

The purposes of this paper are to (a) assess the degree of spatial variability in the surface, cloud and radiative parameters over sea ice using satellite data, (b) evaluate the differences in radiative fluxes calculated from areaaverage surface and cloud properties vs averages of fluxes calculated at a relatively high spatial resolution, and (c) express the averaging error in terms of its effect on sea-ice thickness. The objective is to determine the importance of parameterizing sub-gridcell variability in sea-ice and climate models.

\section{FORMULATION, DATA AND METHODS}

The instantaneous value of a surface radiation-flux quantity $\phi$ at a given point in space and time is a function of the atmospheric forcing $\gamma$ and surface properties $\omega$ :

$$
\phi=f(\gamma, \omega),
$$

where $f$ is a function describing the relationship between $\phi$, $\gamma$ and $\omega$. For the purpose of this study, $\omega$ includes surface forcing terms such as temperature, albedo and surface type, and $\gamma$ includes the atmospheric temperature and humidity profiles, cloud optical depth, cloud fraction, cloud temperature, cloud optical depth, incoming solar radiation at the top of the atmosphere, aerosol amount and ozone amount. The estimate of $\phi$ over an area $A$ is then given by the integration of Equation (1):

$$
\bar{\phi}=\frac{1}{A} \int_{0}^{A} f(\gamma, \omega) \mathrm{d} A .
$$

Since $f$ is inherently micro-scale in nature and both $\gamma$ and $\omega$ may vary in space and time, continuous expressions such as Equation (2) cannot be solved analytically. Unless the relationships between $\phi, \gamma$ and $\omega$ are linear,

$$
\overline{f(\gamma, \omega)} \neq f(\bar{\gamma}, \bar{\omega}),
$$

where the overbar represents an average, i.e. the average flux over some area computed as in Equation (2) is not equal to the flux calculated from the average surface and cloud properties for that area. If three-dimensional effects are not considered, then $\bar{\phi}=\overline{f(\gamma, \omega)}$, which can be calculated from data 
using a discrete form of Equation (2). We refer to this as the "pixel average". A similar concept, the "independent pixel approximation", has been used in studies of three-dimensional radiative transfer effects (cf. Marshak and others, 1998).

The pixel-average concept is employed here to show the magnitude of the inequality in Expression (3). Surface radiative fluxes were computed from satellite-derived surface and cloud properties on a $5 \mathrm{~km}$ pixel scale. These were then averaged over a $255 \times 255 \mathrm{~km}^{2}$ area representing a climatemodel gridcell. The average gridcell flux was also computed from the mean cloud and surface properties within the gridcell. (The mean of the natural logarithm of cloud optical depth was used because of the exponential relationship between it and surface radiation.) We refer to this method as simply the "area average". These two gridcell averages correspond to the left and right sides, respectively, of Expression (3).

Semivariance analysis is employed to describe the spatial and temporal variability of the surface radiative fluxes, cloud and surface properties. The semivariogram is a structure function that, like autocorrelation, describes the correspondence between observations made at some distance or time lag. Therefore, a parameter measured at one location or time provides some information about the parameter at other locations or times. The semivariance $r(h)$ is defined as:

$$
r(h)=\frac{1}{2 N} \sum_{i=1}^{N}\left[\phi\left(x_{i}\right)-\phi\left(x_{i}+h\right)\right]^{2} .
$$

The change in semivariance with lag illustrates how rapidly the autocorrelation changes, while the magnitude of the semivariance indicates the degree of variability. One characteristic value of the semivariogram is the "sill". The sill is the ordinate value at which the semivariogram reaches a maximum and becomes asymptotic, indicating that beyond the corresponding distance (time or space) the variance does not increase substantially.

Data from the Advanced Very High Resolution Radiometer (AVHRR), on board U.S. National Oceanic and Atmospheric Administration polar-orbiting satellites, are used in this study. The specific dataset is a product of the AVHRR Polar Pathfinder (APP) project (Maslanik and others, 1997, 1999, 2000; W. N. Meier and others, 1997, http:// earthinteractions.org). The APP data are twice-daily composites available at $5 \mathrm{~km}$ pixel size for June 1981-98. The study period (September 1997-August 1998) and the area (Fig. 1) encompass the Surface Heat Budget of the Arctic Ocean (SHEBA) field experiment, where an ice-breaker drifted with the pack ice for lyear (Moritz and others, 1993). The APP standard products are clear-sky surface temperature and broadband albedo, a cloud mask, sea-ice motion and the calibrated, geolocated channel data and viewing/illumination geometry. We have extended this product set to include the all-sky surface skin temperature and broadband albedo, cloud properties (particle phase, effective radius, optical depth, temperature and pressure) and radiative fluxes using algorithms in the Cloud and Surface Parameter Retrieval (CASPR) system (Key, 1999). The calculation of cloudy-sky surface skin temperature is based on an empirical relationship between the clear-sky surface skin temperature, wind speed and solar zenith angle (daytime). The cloudy-sky broadband surface albedo is determined using the clear-sky broadband albedo (interpolated from nearby pixels) adjusted by the cloud optical depth and the solar zenith angle. Radiative fluxes are computed in CASPR using FluxNet (Key and

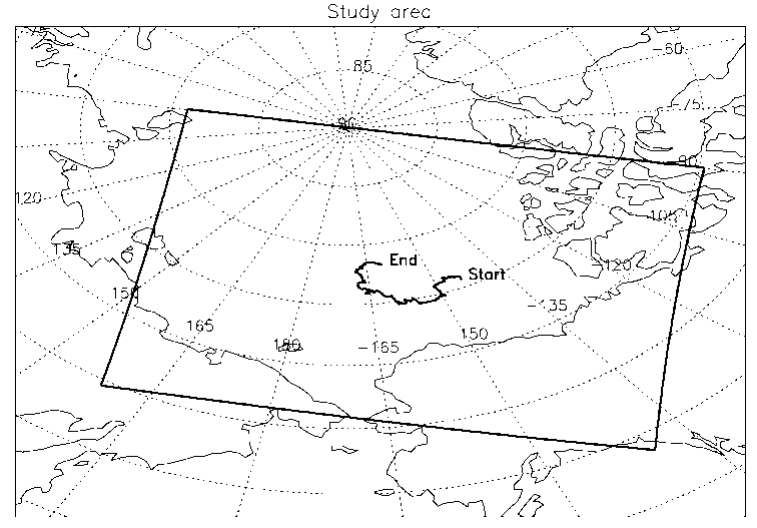

Fig. 1. The study area. The curve is the drift track of SHEBA ship during the year-long experiment. It started at $75.70^{\circ} \mathrm{N}$, $144.10^{\circ} \mathrm{W}$ on 2 October 1997 and ended at $78.20^{\circ} \mathrm{N}$, 160.70 Won 3 August 1998.

Schweiger, 1998). See Key (1999) and references therein for more information on the algorithms and their validation.

\section{TEMPORAL AND SPATIAL VARIABILITY}

Figure 2 shows a time series of AVHRR retrievals for downwelling shortwave and longwave fluxes at the surface (SWD and LWD, respectively), cloud optical depth (TAU) and surface temperature around the SHEBA ship site. The results shown are averages over a $55 \times 55 \mathrm{~km}^{2}$ area centered on the ship and smoothed with a 5 day running-mean filter. The temporal persistence of downwelling short- and longwave fluxes, net radiation flux (NET), cloud fraction, cloud optical depth, surface broadband albedo and surface skin temperature is illustrated as a semivariogram in Figure 3. The semivariance of each parameter was normalized by the maximum semivariance. For both downwelling shortwave and longwave fluxes the sill is reached after 180 days, implying strong temporal correlations. But for cloud fraction and cloud optical depth the persistence is weak, indicating that there is almost no temporal correlation. Another interesting feature is that the net radiation flux at the surface has a different range than that of downwelling shortand longwave fluxes, reaching the sill about after 220 days.

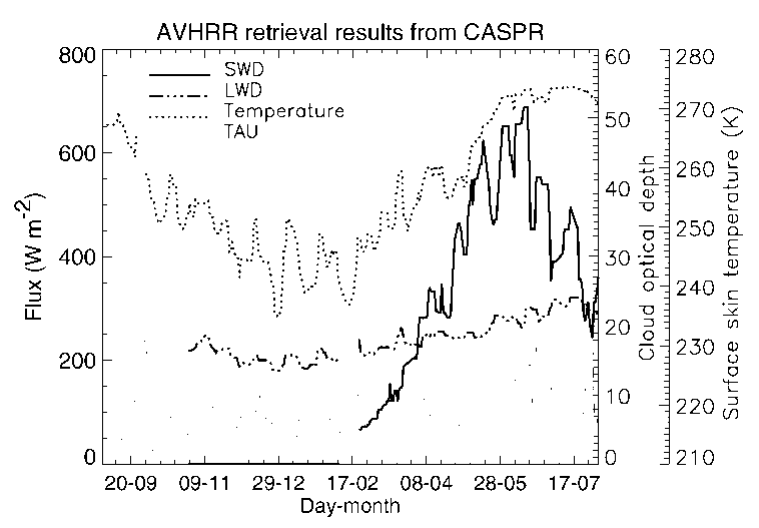

Fig. 2. Time series of downwelling shortwave (SWD) and longwave (LWD) fluxes at the surface, cloud optical depth (TAU) and surface temperature. Values are averages over an area of $55 \times 55 \mathrm{~km}^{2}$ centered on the SHEBA ship and smoothed with a 5 day running mean. 


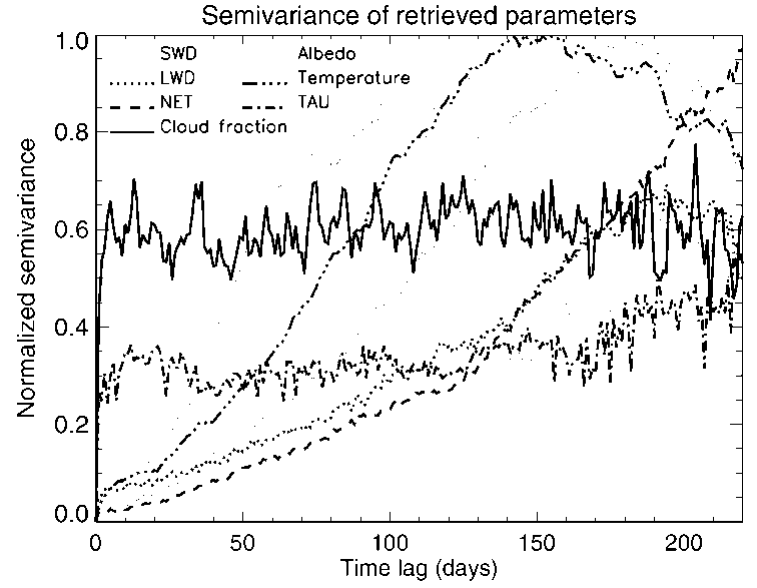

Fig. 3. Time-lag semivariogram for surface downwelling shortand longwave fluxes (SWD and LWD), cloud optical depth (TAU), cloud fraction, surface broadband albedo, surface skin temperature and net radiation at the surface. The semivariance of each parameter was normalized by the maximum semivariance.

Figure 4 shows the spatial semivariogram of surface and cloud properties for June 1998, based on monthly mean values along the longitude $165^{\circ} \mathrm{W}$. Cloud fraction is not shown because sub-pixel cloud fraction was not computed. As with the temporal semivariogram, radiative fluxes, surface skin temperature and surface broadband albedo show persistence with distance. Cloud particle effective radius and optical depth do not, indicating that clouds in this area and time are not horizontally homogeneous as is commonly assumed in sea-ice and climate models. The gradual increase in the normalized semivariance of surface skin temperature and broadband albedo relative to that of cloud optical depth and shortwave radiation implies that the spatial variance increases much less rapidly for surface properties than for cloud properties. An interesting feature of downwelling fluxes and surface albedo is that the semivariograms show sinusoidal wave patterns with spatial scale. Cloud optical depth also shows a similar wave pattern with spatial scale. This phenom-

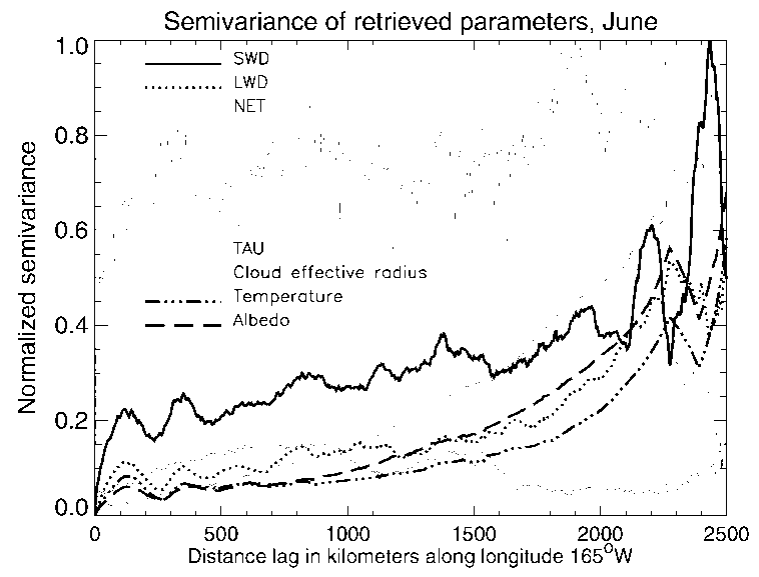

Fig. 4. Space-lag semivariogram for surface downwelling shortand longwave fluxes (SWD and LWD), cloud optical depth (TAU), cloud fraction, surface albedo, surface skin temperature, cloud effective radius, and net radiation flux at the surface. The semivariance of each parameter was normalized by the maximum semivariance. Monthly means along longitude $165^{\circ} \mathrm{W}$ were used.
Table 1. Monthly means of downwelling shortwave (SWD), longwave (LWD) and net (NET) radiation fluxes at the surface $\left(W^{-2}\right)$

\begin{tabular}{ccccccc}
\hline \multicolumn{1}{c}{ Area average over $255 \times 255 \mathrm{~km}^{2}$} & \multicolumn{4}{c}{ Pixel average over $255 \times 255 \mathrm{~km}^{2}$} \\
Month $^{*}$ & SWD & LWD & NET & SWD & LWD & NET \\
& & & & & & \\
\hline \multirow{2}{*}{10} & 0.00 & 218.76 & -11.68 & 0.00 & 223.18 & -13.62 \\
11 & 0.00 & 178.02 & -33.48 & 0.00 & 211.60 & -10.62 \\
12 & 0.00 & 126.31 & -49.59 & 0.00 & 172.14 & -18.13 \\
1 & 0.00 & 204.76 & 2.04 & 0.00 & 203.98 & -1.31 \\
2 & 74.10 & 209.06 & 16.25 & 71.61 & 212.90 & 14.93 \\
3 & 291.51 & 182.67 & 16.28 & 216.08 & 228.82 & 34.16 \\
4 & 494.52 & 234.47 & 60.49 & 465.10 & 237.12 & 51.67 \\
5 & 502.91 & 257.41 & 71.33 & 470.46 & 283.27 & 77.50 \\
6 & 475.58 & 290.04 & 180.08 & 458.41 & 290.01 & 173.19 \\
7 & 258.90 & 295.40 & 124.74 & 243.89 & 301.52 & 118.36 \\
8 & 332.18 & 299.77 & 135.26 & 317.22 & 296.95 & 122.70 \\
& & & & & &
\end{tabular}

* Months 10-12 are in 1997; 1-8 are in 1998. August means are based on only three cases.

enon may be related to synoptic-scale weather systems, at least in June over this part of the Arctic. When such patterns are present, spatial averaging must be done with care.

\section{AREA-AVERAGE AND PIXEL-AVERAGE FLUX BIAS}

Given that (a) surface and cloud parameters exhibit spatial variability on scales less than that of a typical climate- or ice-model gridcell, (b) the magnitude of the variability differs for each parameter, and (c) the relationship between some parameters and surface radiation is non-linear, one would expect that radiative fluxes calculated using average surface and cloud properties over a gridcell would be different from the average of fluxes calculated at some arbitrarily small scale (high resolution) within the cell. In the terminology presented previously, the pixel average for a gridcell must be different from the area average. How different are they for radiative fluxes over sea ice?

Monthly mean downwelling short- and longwave fluxes over $255 \times 255 \mathrm{~km}^{2}$ are given in Table 1 for the two different averaging methods. Table 2 shows both the absolute value of the bias and the percentage bias, where bias is defined as the

Table 2. Monthly mean bias of downwelling shortwave $(S W D)$ and longwave (LWD) radiation fluxes at the surface

\begin{tabular}{|c|c|c|c|c|}
\hline \multirow[b]{2}{*}{ Month* } & \multicolumn{2}{|c|}{ Absolute value over $255 \times 255 \mathrm{~km}^{2}$} & \multicolumn{2}{|c|}{ Percentage over $255 \times 255 \mathrm{~km}^{2}$} \\
\hline & SWD bias & LWD bias & SWD bias & LWD bias \\
\hline 10 & 0.00 & -4.42 & 0.00 & -1.98 \\
\hline 11 & 0.00 & -33.57 & 0.00 & -15.87 \\
\hline 12 & 0.00 & -45.84 & 0.00 & -26.63 \\
\hline 1 & 0.00 & 0.79 & 0.00 & 0.39 \\
\hline 2 & 2.49 & -3.84 & 3.47 & -1.81 \\
\hline 3 & 75.43 & -46.15 & 34.91 & -20.17 \\
\hline 4 & 29.41 & -2.66 & 6.32 & -1.12 \\
\hline 5 & 32.45 & -25.86 & 6.90 & -9.13 \\
\hline 6 & 17.16 & 0.04 & 3.74 & 0.01 \\
\hline 7 & 15.01 & -6.13 & 6.15 & -2.03 \\
\hline 8 & 14.96 & 2.81 & 4.72 & 0.95 \\
\hline
\end{tabular}

\footnotetext{
* Months 10-12 are in 1997; 1-8 are in 1998. August means are based on only three cases. Percentage biases are calculated relative to the pixelaveraged values.
} 


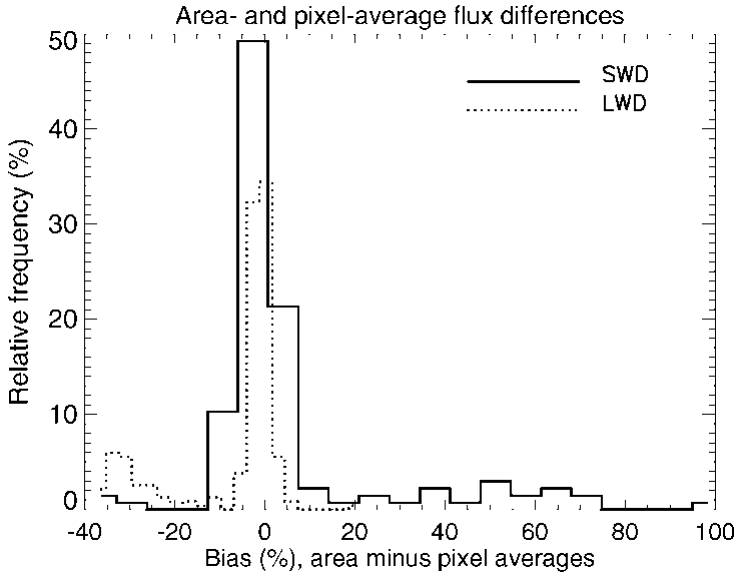

Fig. 5. Relative frequency of flux differences for the area-average and pixel-average flux computations. Values shown are for the period September 1997-August 1998. Downwelling short- and longwave radiation fluxes are denoted by SWD and LWD, respectively.

area-average flux minus the pixel-average flux. Figure 5 shows the frequency of the flux bias for downwelling shortand longwave fluxes. Shortwave biases are generally positive, with a mean annual bias of $9.46 \%$. Longwave flux biases are negative, with a mean annual bias of $-7.04 \%$. The largest monthly mean bias was nearly 35\% in March 1998 for downwelling shortwave flux, and $-27 \%$ for the downwelling longwave flux in December 1997.

The bias introduced by using area-averaged surface and cloud properties to compute surface fluxes can lead to overor underestimates of latent heat, sensible heat and ultimately snow and ice thickness in models. Ebert and Curry (1993; hereafter EB93) evaluated the sensitivity of the equilibrium ice thickness in the Arctic to changes in surface and atmospheric parameters using a one-dimensional sea-ice model. They show that the equilibrium ice thickness changes from $4.3 \mathrm{~m}$ to $1.5 \mathrm{~m}$ for changes in the annual average downwelling shortwave flux of $-5 \%$ to $+5 \%$, where the baseline equilibrium ice thickness is approximately $3 \mathrm{~m}$ (EB93, fig. 15). In the context of the EB93 sensitivity study, our mean annual shortwave bias of $9.46 \%$ implies a decrease in the equilibrium ice thickness to approximately $0.5 \mathrm{~m}$. Regarding the longwave flux bias, the mean annual value of $-7.04 \%$ determined here would increase ice growth, and result in an equilibrium ice thickness of $>10 \mathrm{~m}$ based on the EB93 sensitivity results.

However, there are a number of reasons why the radiative-flux biases may have less impact on ice thickness than the comparisons to EB93 sensitivities imply. First, EB93 show a nearly linear response of ice thickness to changes in the downwelling shortwave flux, but a highly non-linear response to the downwelling longwave flux. The two-dimensional dynamic-thermodynamic model used by Maslanik and Silcox (1993) exhibited a linear response of ice thickness to changes in the shortwave flux, but, unlike EP93, the response to the longwave flux was also linear. They speculate that the different response may be a result of including ice dynamics. Second, the short- and longwave annual flux biases are of opposite sign, and would therefore be offsetting to some degree. Third, flux biases during the freezing season may have a much smaller effect on ice thickness than similar biases in the summer. For example, if the downwelling longwave flux is increased during the winter, the upwelling long- wave and turbulent fluxes may respond such that there is only a modest decrease in ice growth. Conversely, during the summer melt season all of the excess energy would go directly into ice melting, resulting in a large impact on overall thickness (personal communication from G. Maykut, 2000). Fourth, the biases found here were less than the expected uncertainties in the satellite retrievals for some months. In such cases the method used to calculate area-average fluxes may not be important.

\section{GONGLUSIONS}

Satellite retrievals of surface, cloud and radiation parameters over sea ice were used to investigate the spatial and temporal variability of surface and atmospheric parameters in the western Arctic during the SHEBA experiment. Downwelling short- and longwave fluxes at the surface exhibit temporal correlation over a long time period (about 180 days), but cloud optical depth and cloud fraction have nearly no correlation over the time period. Radiative fluxes, surface skin temperature and surface broadband albedo also show persistence with distance, while cloud particle effective radius and optical depth do not. The spatial variance increases much less rapidly for surface properties than for cloud properties.

Given the spatial variability of surface and cloud parameters and, in some cases, their non-linear relationship with radiative fluxes, one would expect that using mean surface and cloud properties within a climate- or ice-model gridcell to compute radiative fluxes could result in substantial errors. This was investigated by computing average radiative fluxes for a $255 \times 255 \mathrm{~km}^{2}$ gridcell as (a) the mean of fluxes calculated for every $5 \mathrm{~km}$ pixel, and (b) the result of using area-average mean surface and cloud properties in the flux calculation. As annual averages the mean shortwave flux bias was $9.46 \%$, while the mean longwave flux bias was $-7.04 \%$. For the SHEBA case this indicates that using mean surface and cloud properties to compute surface radiative fluxes for a gridcell results in an overestimate of the downwelling shortwave flux and an underestimate of the downwelling longwave flux. In terms of ice growth and melt, sensitivity studies done by other investigators imply that the biases observed here may result in substantial errors in the modeled equilibrium ice thickness. Therefore, it is recommended that sub-gridcell inhomogeneity of surface and atmospheric properties be taken into account in sea-ice and climate models.

\section{ACKNOWLEDGEMENTS}

This research was supported by NASA grants NAG5-8625 and NAG5-6666 and U.S. National Science Foundation grant OPP-0096085. We thank C. Fowler, J. Maslanik, T. Scambos and T. Haran for all their work on the APP dataset.

\section{REFERENCES}

Ebert, E. E. and J. A. Curry. 1993. An intermediate one-dimensional thermodynamic sea ice model for investigating ice-atmosphere interactions. $\mathcal{F}$. Geophys. Res., 98 (C6), 10,085-10,109.

Friedl, M. A. 1996. Relationships among remotely sensed data, surface energy balance, and area-averaged fluxes over partially vegetated land surfaces. 7. Appl. Meteorol., 35(11), 2091-2103.

Key, J. 1999. The cloud and surface parameter retrieval (CASPR) system for polar AVHRR. Madison, WI, University of Wisconsin. Cooperative Institute for Meteorological Satellite Studies. 
Key, J. and A. J. Schweiger. 1998. Tools for atmospheric radiative transfer: Streamer and FluxNet. Comput. Geosci., 24(5), 443-451.

Mahrt, L. 1987. Grid-averaged surface fluxes. Mon. Weather Rev., 115(8), 1550-1560.

Marshak, A., S. Davis, R. F. Cahalan and W. Wiscombe. 1998. Nonlocal independent pixel approximation: direct and inverse problems. IEEE Trans. Geosci. Remote Sensing, GE-36(1), 192-205.

Maslanik, J. A. and R. A. Silcox. 1993. Temporal variation of cloud fraction: effects on a simulated sea-ice cover. Geophys. Res. Lett., 20(23), 2651-2654.

Maslanik, J., C. Fowler, J. Key, T. Scambos, T. Hutchinson and W. Emery. 1997. AVHRR-based Polar Pathfinder products for modeling applications. Ann. Glaciol., 25, 388-392.

Maslanik, J., A. Lynch and C. Fowler. 1999. Assessing 2-D and coupled-model simulations of sea ice anomalies using remotely-sensed polar pathfinder products. In Fifth Conference on Polar Meteorology and Oceanography, 10-15 7anuary 1999, Dallas, Texas. Proceedings. Boston, MA, American Meteorological Society, 476-479.

Maslanik, J. A., J. Key, C. W. Fowler, T. Nguyen and X. Wang. In press. Spatial and temporal variability of satellite-derived cloud and surface characteristics during FIRE-ACE. 7. Geophys. Res.

Moritz, R. E., J. A. Curry, A. S. Thorndike and N. Untersteiner. 1993. Surface heat budget of the Arctic Ocean. Seattle, WA, University of Washington. Polar Science Center. Applied Physics Laboratory. SHEBA Project Office. (ARCSS/OAII Tech. Rep 3 .

Sellers, P. 1991. Modeling and observing land-surface-atmosphere interactions on large scales. Surv. Geophys., 21 (1), 85-114. 